\title{
Quantum Potential and Symmetries in Extended Phase Space
}

\author{
Sadollah NASIRI ${ }^{\dagger \ddagger}$ \\ $\dagger$ Department of Physics, Zanjan University, Zanjan, Iran \\ ¥ Institute for Advanced Studies in Basic Sciences, IASBS, Zanjan, Iran \\ E-mail: nasiri@iasbs.ac.ir
}

Received January 06, 2006, in final form May 19, 2006; Published online June 27, 2006

Original article is available at http://www.emis.de/journals/SIGMA/2006/Paper062/

\begin{abstract}
The behavior of the quantum potential is studied for a particle in a linear and a harmonic potential by means of an extended phase space technique. This is done by obtaining an expression for the quantum potential in momentum space representation followed by the generalization of this concept to extended phase space. It is shown that there exists an extended canonical transformation that removes the expression for the quantum potential in the dynamical equation. The situation, mathematically, is similar to disappearance of the centrifugal potential in going from the spherical to the Cartesian coordinates that changes the physical potential to an effective one. The representation where the quantum potential disappears and the modified Hamilton-Jacobi equation reduces to the familiar classical form, is one in which the dynamical equation turns out to be the Wigner equation.
\end{abstract}

Key words: quantum potential; Wigner equation; distribution functions; extended phase space

2000 Mathematics Subject Classification: 81S30

\section{Introduction}

If we assume a polar form for the wave function as the solution of Schrödinger equation, we obtain decomposition of this equation into real and imaginary parts [1,2]. The resulting equations are a pair of coupled partial differential equations in which the amplitude and the phase of the wave function co-determine each other. The real part, is nothing but the familiar HamiltonJacobi equation with a term added that is called the quantum potential, and the imaginary part yields the continuity equation for the amplitude [3]. The quantum potential term in the modified Hamilton-Jacobi equation depends on the magnitude of the wave function rather than its phase. This modification may be equally looked at from the point of view of Newton second law that is modified by the quantum force term. Thus, in the causal interpretation, in addition to the external force, the quantum force, guides the trajectory of the quantum particle in the framework of Bohm's approach to quantum mechanics. Much has been done to identify the quantum aspects of a system within the quantum potential. The concept of quantum internal energy (or stress) as a consequence of the projection from the phase space representation to the configuration space representation was introduced by Takabayasi [4] for the pure states and then extended by Muga et al. [5] to the mixed states. Unlike the classical systems that have kinetic and potential energies, quantum systems also have intrinsic internal energies associated with spatial localization and momentum dispersion emerging from their inherent extended nature. It seems that the quantum potential is a balance between the energies of spatial localization and momentum dispersion in quantum systems suggesting a link to the Heisenberg positionmomentum uncertainty principle [6]. Brown and Hiley [7] showed that the quantum potential 
cannot be dismissed as an artificial term without missing some of the essential novel features of quantum mechanics accounting for the interference, Einstein-Podolsky-Rosen type correlations [8], quantum state teleportation [9], barrier penetration and quantum non-separability [10]. Holland [11], has recently investigated the de Broglie-Bohm law of motion using a variational formulation. He considers a quantum system and suggests a total Lagrangian for the interaction of a point like particle with Schrödinger field without a back reaction of the particle on the wave. The interaction of the particle and the field is attributed to a scalar potential that turns out to be quantum potential, whenever, the constraint of the invariance of Schrödinger equation is applied. The connection of quantum potential with quantum fluctuations and quantum geometry in terms of Weyl curvature has been studied extensively by different authors; see, for example F. and A. Shojai [12], Carroll [13] and the references therein. However, unlike the external potential, the quantum potential is not a pre-assigned function of the system coordinates and can only be derived from the wave function of the system [6] or from the corresponding quantum distribution functions used to calculate the average values of the observables. This representation dependent property of the quantum potential, as the main theme of the present work, allows one to find appropriate representations in which one may obtain desired expressions for the quantum potential that may even be equal to zero. In an interesting work by Carroll [14] it is shown that for a quite general function of position, time and $\hbar, Q(q, t, \hbar)$, there are "generalized" quantum theories having $Q$ as their quantum potential and that the vanishing of the quantum potential depends on the wave function.

Here, we show that in an appropriate phase space representation one may remove the quantum potential from the dynamical equation of a particle in the linear and the harmonic potential keeping the Hamilton-Jacobi equation form invariant. In other words, we show that the quantum potential behaves as a representation dependent quantity in the phase space. The situation seems to be similar to the case of centrifugal potential that disappears in going from spherical to Cartesian coordinate system. The representation is obtained by a canonical transformation on the classical level corresponding to a unitary transformation on the quantum level.

In 1932 the phase space picture of the quantum mechanics was introduced by the pioneering work of Wigner [15] and extended by Moyal [16], Hillary et al. [17], Mehta [18], Agarwal and Wolf [19], Han et al. [20] Kim and Wigner [21] and Jannussis et al. [22], and many others. Although, the arguments invoked on the basis of the Heisenberg uncertainty principle made the physical meaning of the "phase space points" problematic, things have changed and phase space techniques mainly formulated by the theory of deformation quantization [23] and a family of Schrödinger equations in phase space [24, 25, 26, 27, 28] are now widely accepted and used. As an approach of the latter type, Sobouti and Nasiri [29] have proposed a formulation of quantum statistical mechanics by generalizing the principle of least action to the trajectories in phase space, and a canonical quantization procedure in this space. In the extended phase space (EPS) formalism, positions and momenta are assumed to be independent c-number variables. Therefore, the canonical transformations allow one to define different phase space representations for quantum distribution functions with their corresponding associated ordering rules. As a special case, we obtain the Wigner distribution functions $[16,18]$ by a simple extended canonical transformation that corresponds to an extended unitary transformation at quantum level.

Using the EPS method, we look for a canonical transformation that removes the quantum potential in the dynamical equation. In Section 2, the EPS formalism that treats the momentum and position coordinates in a symmetric way after quantization, is reviewed. In Section 3, the extended canonical transformations are introduced and are used to obtain the Wigner equation and the corresponding Wigner functions. In Section 4, the quantum potential is written in both configuration and momentum space representations for linear and harmonic potentials. It is argued that the form of the quantum potential in the momentum space is not, in general, as simple as that of the configuration space. Furthermore, an expression for the quantum potential 
is obtained in EPS. In Section 5, an appropriate phase space representation, which is shown to be the Wigner representation of quantum mechanics in the phase space [15], is found in which the quantum potential is removed in dynamical equations for linear and harmonic potentials. Finally, Section 6 is devoted to the concluding remarks.

\section{Review of EPS formalism}

A direct approach to quantum statistical mechanics is proposed by extending the conventional phase space and by applying the canonical quantization procedure to the extended quantities in this space. Here, a brief review of this formalism is presented. For more details, see Sobouti and Nasiri [29].

Let $\mathcal{L}^{q}(q, \dot{q})$ be a Lagrangian specifying a system in $q$ space. A trajectory of the system in this space is obtained by solving the Euler-Lagrange equations for $q(t)$,

$$
\frac{d}{d t} \frac{\partial \mathcal{L}^{q}}{\partial \dot{q}}-\frac{\partial \mathcal{L}^{q}}{\partial q}=0
$$

The derivative $\frac{\partial \mathcal{L}^{q}}{\partial \dot{q}}$ calculated on an actual trajectory, that is, on a solution of equation (1), is the momentum $p$ conjugate to $q$. The same derivative calculated on a virtual orbit, not a solution of equation (1), exists. It may not, however, be interpreted as a canonical momentum. Let $H(p, q)$ be a function in phase space which is the Hamiltonian of the system, whenever $p$ and $q$ are canonical pairs. It is related to $\mathcal{L}^{q}$ through the Legendre transformation,

$$
H\left(\frac{\partial \mathcal{L}^{q}}{\partial \dot{q}}, q\right)=\dot{q} \frac{\partial \mathcal{L}^{q}}{\partial \dot{q}}-\mathcal{L}^{q}(q, \dot{q})
$$

For a given $\mathcal{L}^{q}$, equation (2) is an algebraic equation for $H$. One may, however, take a different point of view. For a given functional form of $H(p, q)$, equation (2) may be considered as a differential equation for $\mathcal{L}^{q}$. Its non unique solutions differ from one another by total time derivatives. One may also study the same system in the momentum space. Let $\mathcal{L}^{p}(p, \dot{p})$ be a Lagrangian in $p$ space [30]. It is related to $H(p, q)$ as follows,

$$
H\left(p, \frac{\partial \mathcal{L}^{p}}{\partial \dot{p}}\right)=-\dot{p} \frac{\partial \mathcal{L}^{p}}{\partial \dot{p}}+\mathcal{L}^{p}(p, \dot{p}) .
$$

Here the functional dependence of $H$ on its argument is the same as in equation (2). In principle, equation (3) should be solvable for $\mathcal{L}^{p}$ up to an additive total time derivative term. Once $\mathcal{L}^{p}$ is known the actual trajectories in $p$ space are obtainable from an Euler-Lagrange equation analogous to equation (1) in which $q$ is replaced by $p$. That is

$$
\frac{d}{d t} \frac{\partial \mathcal{L}^{p}}{\partial \dot{p}}-\frac{\partial \mathcal{L}^{p}}{\partial p}=0
$$

The derivative $\frac{\partial \mathcal{L}^{p}}{\partial \dot{p}}$ along an actual $p$ trajectory is the canonical coordinate conjugate to $p$. Calculated on a virtual orbit, it is not.

A formulation of quantum statistical mechanics is possible by extending the conventional phase space and by applying the canonical quantization procedure to the extended quantities in this space. Assuming the phase space coordinates $p$ and $q$ to be independent variables on the virtual trajectories, allows one to define momenta $\pi_{p}$ and $\pi_{q}$, conjugate to $p$ and $q$, respectively. One may combine the two pictures and define an extended Lagrangian in the phase space as the sum of $p$ and $q$ Lagrangians,

$$
\mathcal{L}(p, q, \dot{p}, \dot{q})=-\dot{p} q-\dot{q} p+\mathcal{L}^{p}(p, \dot{p})+\mathcal{L}^{q}(q, \dot{q}) .
$$


The first two terms in equation (4) constitute a total time derivative. The equations of motion are

$$
\begin{aligned}
& \frac{d}{d t} \frac{\partial \mathcal{L}}{\partial \dot{p}}-\frac{\partial \mathcal{L}}{\partial p}=\frac{d}{d t} \frac{\partial \mathcal{L}^{p}}{\partial \dot{p}}-\frac{\partial \mathcal{L}^{p}}{\partial p}=0 \\
& \frac{d}{d t} \frac{\partial \mathcal{L}}{\partial \dot{q}}-\frac{\partial \mathcal{L}}{\partial q}=\frac{d}{d t} \frac{\partial \mathcal{L}^{q}}{\partial \dot{q}}-\frac{\partial \mathcal{L}^{q}}{\partial q}=0
\end{aligned}
$$

The $p$ and $q$ in equations (4) and (5) are not, in general, canonical pairs. They are so only on actual trajectories and through a proper choice of the initial values. This gives the freedom of introducing a second set of canonical momenta for both $p$ and $q$. One does this through the extended Lagrangian. Thus

$$
\pi_{p}=\frac{\partial \mathcal{L}}{\partial \dot{p}}=\frac{\partial \mathcal{L}^{p}}{\partial \dot{p}}-q, \quad \pi_{q}=\frac{\partial \mathcal{L}}{\partial \dot{q}}=\frac{\partial \mathcal{L}^{q}}{\partial \dot{q}}-p .
$$

Evidently, $\pi_{p}$ and $\pi_{q}$ vanish on actual trajectories and remain non zero on virtual ones. From these extended momenta, one defines an extended Hamiltonian,

$$
\begin{aligned}
\mathcal{H}\left(\pi_{p}, \pi_{q}, p, q\right) & =\dot{p} \pi_{p}+\dot{q} \pi_{q}-\mathcal{L}=H\left(p+\pi_{q}, q\right)-H\left(p, q+\pi_{p}\right) \\
& =\sum \frac{1}{n !}\left\{\left(\frac{\partial^{n} H}{\partial p^{n}}\right)_{\left.\right|_{\pi_{q}=0}} \pi_{q}^{n}-\left(\frac{\partial^{n} H}{\partial q^{n}}\right)_{\left.\right|_{\pi_{p}=0}} \pi_{p}^{n}\right\} .
\end{aligned}
$$

By means of utilisation of the canonical quantization rule, the following postulates are outlined:

a) Let $p, q, \pi_{p}$ and $\pi_{q}$ be operators in a Hilbert space, $\mathbf{X}$, of all complex functions, satisfying the following commutation relations

$$
\begin{aligned}
& {\left[\pi_{q}, q\right]=-i \hbar, \quad \pi_{q}=-i \hbar \frac{\partial}{\partial q},} \\
& {\left[\pi_{p}, p\right]=-i \hbar, \quad \pi_{p}=-i \hbar \frac{\partial}{\partial p}, \quad[p, q]=\left[\pi_{p}, \pi_{q}\right]=0 .}
\end{aligned}
$$

By virtue of equations (7), the extended Hamiltonian, $\mathcal{H}$, will also be an operator in $\mathbf{X}$.

b) A state function $\chi(p, q, t) \in \mathbf{X}$ is assumed to satisfy the following dynamical equation

$$
\begin{aligned}
i \hbar \frac{\partial \chi}{\partial t}=\mathcal{H} \chi & =\left[H\left(p-i \hbar \frac{\partial}{\partial q}, q\right)-H\left(p, q-i \hbar \frac{\partial}{\partial p}\right)\right] \chi \\
& =\sum \frac{(-i \hbar)^{n}}{n !}\left\{\frac{\partial^{n} H}{\partial p^{n}} \frac{\partial^{n}}{\partial q^{n}}-\frac{\partial^{n} H}{\partial q^{n}} \frac{\partial^{n}}{\partial p^{n}}\right\} \chi .
\end{aligned}
$$

The evolution operator on the right hand side of equation (8) is Hermitian as far as the Hamiltonian $H$ is Hermitian and the extension operation is done by the canonical transformations corresponding to the unitary transformations at quantum level. Otherwise, it is not Hermitian and the evolution as described is not unitary. In this case one may wonder that the probability conservation may be violated. However, it is known that $\chi$, unlike the classical Liouville equation, is not a probability distribution function. It may become negative or even complex in some regions of the phase space.

c) The averaging rule for an observable $O(p, q)$, a $c$-number operator in this formalism, is given as

$$
\langle O(p, q)\rangle=\int O(p, q) \chi^{*}(p, q, t) d p d q .
$$


To find the solutions for equation (8) one may assume

$$
\chi(p, q, t)=F(p, q, t) e^{-i p q / \hbar} .
$$

The phase factor comes out due to the total derivative in the Lagrangian of equation (4), $-d(p q) / d t$. The effect is the appearance of a phase factor, $\exp (-i p q / \hbar)$, in the state function that would have been in the absence of the total derivative. It is easily verified that

$$
\left(p-i \hbar \frac{\partial}{\partial q}\right) \chi=i \hbar \frac{\partial F}{\partial q} e^{-i p q / \hbar}, \quad\left(q-i \hbar \frac{\partial}{\partial p}\right) \chi=i \hbar \frac{\partial F}{\partial p} e^{-i p q / \hbar} .
$$

Substituting of equations (10) into equation (8) and eliminating of the exponential factor gives

$$
H\left(-i \hbar \frac{\partial}{\partial q}, q\right)-H\left(p,-i \hbar \frac{\partial}{\partial p}\right) F=i \hbar \frac{\partial F}{\partial t} .
$$

Equation (11) has separable solutions of the form

$$
F(p, q, t)=\psi(q, t) \phi^{*}(p, t)
$$

where $\psi(q, t)$ and $\phi(p, t)$ are the solutions of the Schrödinger equation in $q$ and $p$ representations, respectively. The solution of the form (9) associated with anti-standard ordering rule satisfies equation (8) and is one possible distribution function. For more details on the admissibility of the above distribution function, their interesting properties and the correspondence rules, the interested reader may consult Hillary et al. [17] and Sobouti and Nasiri [29].

\section{The extended canonical transformations}

The canonical transformations that keeps the extended Hamilton equation of motion form invariant are possible in EPS [29]. Consider an infinitesimal extended canonical transformation on $p, q, \pi_{p}, \pi_{q}$ as follows

$$
p \rightarrow p+\alpha \pi_{q}, \quad q \rightarrow q+\beta \pi_{p}, \quad \pi_{p} \rightarrow \pi_{p}+\gamma q, \quad \pi_{q} \rightarrow \pi_{q}+\eta p .
$$

The above transformation is prepared in such a way that the ordering problem does not exist according to the commutation relations of equation (7). The transformation to be canonical, one must have [30]

$$
-\beta+\alpha=0, \quad-\beta \eta+1=1, \quad-1+\alpha \gamma=-1 .
$$

The result is $\beta=\alpha$ and $\eta=\gamma=0$. The corresponding generator is [31]

$$
G=\pi_{p} \pi_{q}=-\hbar^{2} \frac{\partial^{2}}{\partial p \partial q} .
$$

Then, the unitary transformation for finite $\alpha$ becomes

$$
U=e^{\alpha G / i \hbar}=e^{-i \alpha \hbar \partial^{2} / \partial p \partial q}=e^{-i \alpha \hbar \partial^{2} / \partial P \partial Q}, \quad U U^{\dagger}=1 .
$$

By means of utilisation of equations (6) and (14) for $\alpha=-1 / 2$, the new extended Hamiltonian becomes

$$
\mathcal{H}^{\prime}=U \mathcal{H} U^{-1}=H\left(p+\frac{1}{2} \pi_{q}, q-\frac{1}{2} \pi_{p}\right)-H\left(p-\frac{1}{2} \pi_{q}, q+\frac{1}{2} \pi_{p}\right) .
$$


For $H=\frac{1}{2 m} p^{2}+V(q)$, equation (15) gives

$$
\mathcal{H}^{\prime}=\frac{1}{2 m}\left(p+\frac{1}{2} \pi_{q}\right)^{2}+V\left(q-\frac{1}{2} \pi_{p}\right)-\frac{1}{2 m}\left(p-\frac{1}{2} \pi_{q}\right)^{2}-V\left(q+\frac{1}{2} \pi_{p}\right) .
$$

As explicit examples we examine the above transformation for a particle in the linear and harmonic potentials. For the linear potential, $H=\frac{1}{2 m} p^{2}+b q, b$ a constant, one gets simply

$$
\mathcal{H}^{\prime}=\frac{p}{m} \pi_{q}-b \pi_{p}
$$

while, for harmonic potential, $H=\frac{1}{2 m} p^{2}+\frac{1}{2} k q^{2}, k=m \omega^{2}$, one gets simply

$$
\mathcal{H}^{\prime}=\frac{p}{m} \pi_{q}-k q \pi_{p}
$$

By means of utilisation of equation (16), the evolution equation (8) and the distribution functions $\chi(p, q, t)$ transform to

$$
i \hbar \frac{\partial W}{\partial t}=\mathcal{H}^{\prime} W=-i \hbar \frac{p}{m} \frac{\partial W}{\partial q}+\sum \frac{i \hbar}{(2 n+1) !}\left(\frac{\hbar}{2 i}\right)^{2 n} \frac{\partial^{2 n+1} V}{\partial q^{2 n+1}} \frac{\partial^{2 n+1} W}{\partial p^{2 n+1}}
$$

and

$$
W(p, q, t)=U \chi=\int \psi\left(q+\frac{1}{2} \hbar \tau, t\right) \psi^{*}\left(q-\frac{1}{2} \hbar \tau, t\right) e^{-i p \tau} d \tau,
$$

respectively. Equation (19a) is the Wigner equation governing the Wigner distribution functions given by equation (19b) [13]. Therefore, we call the new form of the EPS obtained by transformation (13) on the old one for $\beta=\alpha=-\frac{1}{2}$ as the Wigner representation.

\section{Quantum potential and generalization to the EPS}

a) Quantum potential in $\boldsymbol{q}$ space. Let the Schrödinger wave function in $q$ space be written as follows [1]

$$
\psi(q, t)=R^{q}(q, t) e^{i S^{q}(q, t) / \hbar},
$$

where $R^{q}(q, t)$ is the amplitude and $S^{q}(q, t)$ is the action that serves as the phase of the wave function in $q$ space and is

$$
S^{q}(q, t)=\int^{t} \mathcal{L}^{q}\left(q, \dot{q}, t^{\prime}\right) d t^{\prime}
$$

Using equation (20) in the Schrödinger equation one gets

$$
\begin{aligned}
& i \hbar\left(\frac{\partial R^{q}}{\partial t}+\frac{i}{\hbar} R^{q} \frac{\partial S^{q}}{\partial t}\right) \\
& \quad=-\frac{\hbar^{2}}{2 m}\left[\frac{\partial^{2} R^{q}}{\partial q^{2}}+\frac{2 i}{\hbar} \frac{\partial R^{q}}{\partial q} \frac{\partial S^{q}}{\partial q}+\frac{i}{\hbar} R^{q} \frac{\partial^{2} S^{q}}{\partial q^{2}}-\frac{1}{\hbar^{2}} R^{q}\left(\frac{\partial S^{q}}{\partial q}\right)^{2}\right]+V(q) R^{q} .
\end{aligned}
$$

The real part of equation (22) gives

$$
\frac{\partial S^{q}}{\partial t}-\frac{\hbar^{2}}{2 m} \frac{1}{R^{q}} \frac{\partial^{2} R^{q}}{\partial q^{2}}+\frac{1}{2 m}\left(\frac{\partial S^{q}}{\partial q}\right)^{2}+V(q)=0 .
$$


Apart from the term $-\frac{\hbar^{2}}{2 m} \frac{1}{R^{q}} \frac{\partial^{2} R^{q}}{\partial q^{2}}$, equation (23) for $p=\frac{\partial S^{q}}{\partial q}$ is the familiar Hamilton-Jacobi equation. The term $-\frac{\hbar^{2}}{2 m} \frac{1}{R^{q}} \frac{\partial^{2} R^{q}}{\partial q^{2}}$ is known as the quantum potential [1]. The imaginary part of equation (22) yields a continuity equation for $|R|^{2}$, that is not our interest here.

b) Quantum potential in $\boldsymbol{p}$ space. The expression for quantum potential in $p$ space does not, in general, have a simple one term form as in equation (22) obtained for $q$ space [6]. However, the cases of linear and harmonic potentials are relatively simple. Potentials of higher polynomial order generate series of terms having even powers of $\hbar^{2}$. See [6] for some explicit examples. Here, we concentrate on the cases of linear and harmonic potentials.

i) Linear potential. The Schrödinger equation for a particle in a linear potential of $V(q)=b q$ in $p$ representation is

$$
i \hbar \frac{\partial \phi(p, t)}{\partial t}=\frac{p^{2}}{2 m} \phi(p, t)+i \hbar b \frac{\partial \phi(p, t)}{\partial p},
$$

where $\phi(p, t)$ is the Schrödinger wave function in $p$ space and is assumed to be

$$
\phi(p, t)=R^{p}(p, t) e^{-i S^{p}(p, t) / \hbar},
$$

where $R^{p}(p, t)$ is the amplitude and $S^{p}(p, t)$ is defined as

$$
S^{p}(p, t)=\int^{t} \mathcal{L}^{p}\left(p, \dot{p}, t^{\prime}\right) d t^{\prime}
$$

where $\mathcal{L}^{p}$ is given by equation (3).

Substituting of equation (25) into (24) gives

$$
i \hbar\left(\frac{\partial R^{p}}{\partial t}+\frac{i}{\hbar} R^{p} \frac{\partial S^{p}}{\partial t}\right)=\frac{p^{2}}{2 m} R^{p}+i \hbar b\left(\frac{\partial R^{p}}{\partial p}+\frac{i R^{p}}{\hbar} \frac{\partial S^{p}}{\partial p}\right) .
$$

The real part of equation (27) gives

$$
\frac{\partial S^{p}}{\partial t}+\frac{p^{2}}{2 m}-b \frac{\partial S^{p}}{\partial p}=0 .
$$

Identifying $q=-\frac{\partial S^{p}}{\partial p}$, gives

$$
\frac{\partial S^{p}}{\partial t}+H=0 .
$$

Note that, unlike the $q$ space, there is no quantum potential term for the linear potential in $p$ space [6]. In other words, the quantum potential is removed in going from the $q$ space to the $p$ space. Thus, if one works out the problem of a particle in the linear potential in the $p$ space, quantum potential will not appear in the dynamical equations.

ii) Harmonic potential. By the same procedure followed in part (i), one gets for harmonic potential $V(q)=\frac{1}{2} k q^{2}$,

$$
\begin{aligned}
& i \hbar\left(\frac{\partial R^{p}}{\partial t}+\frac{i}{\hbar} R^{p} \frac{\partial S^{p}}{\partial t}\right) \\
& \quad=\frac{p^{2}}{2 m} R^{p}-\frac{\hbar^{2}}{2} k\left[\frac{\partial^{2} R^{p}}{\partial p^{2}}+\frac{2 i}{\hbar} \frac{\partial R^{p}}{\partial p} \frac{\partial S^{p}}{\partial p}+\frac{i}{\hbar} R^{p} \frac{\partial^{2} S^{p}}{\partial p^{2}}-\frac{1}{\hbar^{2}} R^{p}\left(\frac{\partial S^{p}}{\partial p}\right)^{2}\right]
\end{aligned}
$$

The real part of equation (28) gives

$$
\frac{\partial S^{p}}{\partial t}+\frac{p^{2}}{2 m}-\frac{\hbar^{2}}{2} \frac{k}{R^{p}} \frac{\partial^{2} R^{p}}{\partial p^{2}}+\frac{1}{2} k\left(\frac{\partial S^{p}}{\partial p}\right)^{2}=0 .
$$


Equation (29) is the modified Hamilton-Jacobi equation for the harmonic potential in the $p$ space and contains an extra term, $-\frac{\hbar^{2}}{2} \frac{k}{R^{p}} \frac{\partial^{2} R^{p}}{\partial p^{2}}$, as $p$ space version of the quantum potential.

c) Quantum potential in the EPS. To generalize the concept of quantum potential into the EPS we again consider the cases of the linear and harmonic potentials as we did in part $\mathbf{b}$.

i) Linear potential. The extended Hamiltonian of equation (6) for the linear potential becomes

$$
\mathcal{H}=\frac{\pi_{q}^{2}}{2 m}+\frac{p}{m} \pi_{q}-b \pi_{p}
$$

The state function, $\chi$, in equation (8) is, in general, a complex function. Thus, we assume

$$
\chi(p, q, t)=\mathcal{R}(p, q, t) e^{i \mathcal{S}(p, q, t) / \hbar},
$$

where $\mathcal{R}(p, q, t)$ is the amplitude and $\mathcal{S}(p, q, t)$ is the phase given by

$$
\mathcal{S}(p, q, t)=\int^{t} \mathcal{L}\left(p, q, \dot{p}, \dot{q}, t^{\prime}\right) d t^{\prime}
$$

where $\mathcal{L}(p, q, \dot{p}, \dot{q}, t)$ is defined by equation (4).

Using equation (4), one gets

$$
\mathcal{S}(p, q, t)=S^{p}+S^{q}-p q,
$$

where $S^{q}$ and $S^{p}$ are given by equations (21) and (26), respectively. An alternative approach to equation (31) is to use equations (20) and (25) in equations (9) and (12) and compare the result with equation (30). One may also see that $\mathcal{R}(p, q, t)=R^{q}(q, t) R^{* p}(p, t)$.

Equation (8) now gives

$$
\begin{aligned}
i \hbar\left(\frac{\partial \mathcal{R}}{\partial t}+\frac{i \mathcal{R}}{\hbar} \frac{\partial \mathcal{S}}{\partial t}\right)= & -\frac{\hbar^{2}}{2 m}\left[\frac{\partial^{2} \mathcal{R}}{\partial q^{2}}+\frac{2 i}{\hbar} \frac{\partial \mathcal{R}}{\partial q} \frac{\partial \mathcal{S}}{\partial q}+\frac{i \mathcal{R}}{\hbar} \frac{\partial^{2} \mathcal{S}}{\partial q^{2}}-\frac{\mathcal{R}}{\hbar^{2}}\left(\frac{\partial \mathcal{S}}{\partial q}\right)^{2}\right] \\
& -\frac{i \hbar p}{m}\left(\frac{\partial \mathcal{R}}{\partial q}+\frac{i \mathcal{R}}{\hbar} \frac{\partial \mathcal{S}}{\partial q}\right)+i \hbar b\left(\frac{\partial \mathcal{R}}{\partial p}+\frac{i \mathcal{R}}{\hbar} \frac{\partial \mathcal{S}}{\partial p}\right) .
\end{aligned}
$$

With assumption

$$
\pi_{p}=\frac{\partial \mathcal{S}(p, q, t)}{\partial p} \quad \text { and } \quad \pi_{q}=\frac{\partial \mathcal{S}(p, q, t)}{\partial q},
$$

the real part of equation (32) gives

$$
\frac{\partial \mathcal{S}}{\partial t}-\frac{\hbar^{2}}{2 m} \frac{1}{\mathcal{R}} \frac{\partial^{2} \mathcal{R}}{\partial q^{2}}+\mathcal{H}=0
$$

Equation (33) is the modified Hamilton-Jacobi equation for the linear potential in EPS.

ii) Harmonic potential. The extended Hamiltonian for harmonic potential is

$$
\mathcal{H}=\frac{\pi_{q}^{2}}{2 m}+\frac{p}{m} \pi_{q}-\frac{1}{2} k \pi_{p}^{2}-k q \pi_{p} .
$$

The corresponding evolution equation becomes

$$
i \hbar\left(\frac{\partial \mathcal{R}}{\partial t}+\frac{i \mathcal{R}}{\hbar} \frac{\partial \mathcal{S}}{\partial t}\right)=-\frac{\hbar^{2}}{2 m}\left[\frac{\partial^{2} \mathcal{R}}{\partial q^{2}}+\frac{2 i}{\hbar} \frac{\partial \mathcal{R}}{\partial q} \frac{\partial \mathcal{S}}{\partial q}+\frac{i \mathcal{R}}{\hbar} \frac{\partial^{2} \mathcal{S}}{\partial q^{2}}-\frac{\mathcal{R}}{\hbar^{2}}\left(\frac{\partial \mathcal{S}}{\partial q}\right)^{2}\right]
$$




$$
\begin{aligned}
& -\frac{i \hbar p}{m}\left(\frac{\partial \mathcal{R}}{\partial q}+\frac{i \mathcal{R}}{\hbar} \frac{\partial \mathcal{S}}{\partial q}\right)+\frac{k \hbar^{2}}{2}\left[\frac{\partial^{2} \mathcal{R}}{\partial p^{2}}+\frac{2 i}{\hbar} \frac{\partial \mathcal{R}}{\partial p} \frac{\partial \mathcal{S}}{\partial p}+\frac{i \mathcal{R}}{\hbar} \frac{\partial^{2} \mathcal{S}}{\partial p^{2}}-\frac{\mathcal{R}}{\hbar^{2}}\left(\frac{\partial \mathcal{S}}{\partial p}\right)^{2}\right] \\
& +i \hbar k q\left(\frac{\partial \mathcal{R}}{\partial p}+\frac{i \mathcal{R}}{\hbar} \frac{\partial \mathcal{S}}{\partial p}\right) .
\end{aligned}
$$

The real part of equation (34) gives

$$
\frac{\partial \mathcal{S}}{\partial t}-\frac{\hbar^{2}}{2 m} \frac{1}{\mathcal{R}} \frac{\partial^{2} \mathcal{R}}{\partial q^{2}}+\frac{\hbar^{2} k}{2} \frac{1}{\mathcal{R}} \frac{\partial^{2} \mathcal{R}}{\partial p^{2}}+\mathcal{H}=0
$$

Equation (35) is the modified Hamilton-Jacobi equation for harmonic potential in EPS. The second and the third terms in equation (35), together, define the quantum potential in extended phase space. The second term is the EPS counterpart of quantum potential in $q$ space and the third term is the same thing in $p$ space.

\section{How to remove the quantum potential}

We look for a possible extended canonical transformation that could remove the quantum potential from the modified Hamilton-Jacobi equations (33) and (35). In this respect we consider the following linear transformation

$$
p \rightarrow p+\alpha \pi_{q}, \quad q \rightarrow q+\alpha \pi_{p}, \quad \pi_{p} \rightarrow \pi_{p}, \quad \pi_{q} \rightarrow \pi_{q} .
$$

As argued in Section 3, transformation (36) is canonical for any arbitrary $\alpha$. Thus, the new extended Hamiltonians for the linear and the harmonic potentials become

$$
\mathcal{H}^{\prime}=\frac{\pi_{q}^{2}}{2 m}+\frac{\left(p+\alpha \pi_{q}\right)}{m} \pi_{q}-b \pi_{p}
$$

and

$$
\mathcal{H}^{\prime}=\frac{\pi_{q}^{2}}{2 m}+\frac{\left(p+\alpha \pi_{q}\right)}{m} \pi_{q}-\frac{1}{2} k \pi_{p}^{2}-k\left(q+\alpha \pi_{p}\right) \pi_{p}
$$

respectively.

It can be easily shown that the modified Hamilton-Jacobi equations (33) and (35) transform to

$$
\frac{\partial \mathcal{S}^{\prime}}{\partial t}-\frac{\hbar^{2}}{m}\left(\frac{1}{2}+\alpha\right) \frac{1}{\mathcal{R}^{\prime}} \frac{\partial^{2} \mathcal{R}^{\prime}}{\partial q^{2}}+\frac{p}{m} \pi_{q}-b \pi_{p}=0
$$

and

$$
\frac{\partial \mathcal{S}^{\prime}}{\partial t}-\frac{\hbar^{2}}{m}\left(\frac{1}{2}+\alpha\right) \frac{1}{\mathcal{R}^{\prime}} \frac{\partial^{2} \mathcal{R}^{\prime}}{\partial q^{2}}+\hbar^{2} k\left(\frac{1}{2}+\alpha\right) \frac{1}{\mathcal{R}^{\prime}} \frac{\partial^{2} \mathcal{R}^{\prime}}{\partial p^{2}}+\frac{p}{m} \pi_{q}-k q \pi_{p}=0
$$

respectively.

With assumption $\alpha=-\frac{1}{2}$ and by means of utilisation of equations (17) and (18), both of equations (37) and (38) simultaneously become

$$
\frac{\partial \mathcal{S}^{\prime}}{\partial t}+\mathcal{H}^{\prime}=0
$$

Equation (39) has the familiar form of classical Hamilton-Jacobi equation. Note that, as claimed before, equation (13) transforms equation (8) into Wigner equation for $\alpha=-\frac{1}{2}$ and $\eta=\gamma=0$. 
Thus, we conclude that equation (39) in which the quantum potential is disappeared is obtained in the Wigner representation. The generalization of the present technique to the potentials having a multinomial expansion is under consideration. So far, it is already shown that one may take away the quantum potential from the modified Hamilton-Jacobi equation to the leading terms proportional to the higher powers of $\hbar$ and remove the quantum potential up to the desired approximation order.

\section{Conclusions}

In this paper the concept of quantum potential in configuration space is generalized to the momentum space as well as the phase space. The modified Hamilton-Jacobi equation containing the quantum potential is obtained in these spaces by substituting a polar form of the distribution function into the corresponding dynamical equation. By means of utilisation of the EPS formalism, it is shown that for the cases of linear and harmonic potentials one may remove the quantum potential from the modified Hamilton-Jacobi equation and preserve its standard form as in classical mechanics. The Wigner representation in phase space which is obtained by a canonical transformation in EPS is found to be the appropriate representation in which the quantum potential is removed for these potentials. Generalization of the technique for arbitrary potentials is being under consideration.

\section{A Appendix}

For a given Hamiltonian $H(p, q)$ one may consider equation (2) as a differential equation for $\mathcal{L}^{q}$. For harmonic potential,

$$
H(p, q)=\frac{1}{2 m} p^{2}+\frac{1}{2} k q^{2}, \quad k=m \omega^{2},
$$

one obtains

$$
\mathcal{L}^{q}(q, \dot{q})=\frac{1}{2} m \dot{q}^{2}-\frac{1}{2} k q^{2},
$$

as the solution. In the same manner, using equation (3), one obtains

$$
\mathcal{L}^{p}(p, \dot{p})=\frac{p^{2}}{2 m}-\frac{1}{2 k} \dot{p}^{2}
$$

as a possible solution. Using Euler-Lagrange equation in the $q$ space, one gets,

$$
m \ddot{q}=-k q .
$$

For an initial condition, say,

$$
q(0)=0, \quad \dot{q}(0)=\dot{q}_{0},
$$

the solution is

$$
q(t)=\frac{1}{\omega} \dot{q}_{0} \sin (\omega t) \quad \text { and } \quad \dot{q}(t)=\dot{q}_{0} \cos (\omega t) .
$$

For arbitrary initial conditions the solution in the $p$ space is independent of the solution in the $q$ space. However, in order to describe the same oscillator solution, the initial conditions assumed 
for the oscillator in the $q$ space must be translated into the $p$ space. This can be done using the relations that instantaneously couples the $q$ space variables to those in the $p$ space, i.e.,

$$
p=\frac{\partial \mathcal{L}^{q}}{\partial \dot{q}}=m \dot{q}
$$

which is the definition of the canonical momentum (here, the same as the mechanical momentum) and

$$
q=\frac{\partial \mathcal{L}^{p}}{\partial \dot{p}}=-\frac{\dot{p}}{k} \quad \text { or } \quad \dot{p}=-k q,
$$

which is Newton law of motion. Thus, one obtains

$$
p(0)=m \dot{q}_{0} \quad \text { and } \quad \dot{p}(0)=0,
$$

as initial conditions and

$$
p=m \dot{q}_{0} \cos \omega t, \quad \dot{p}=-m \omega \dot{q}_{0} \sin \omega t,
$$

as the solutions in the $p$ space describing the same oscillator in the $q$ space. The same way could be followed for linear potential while the variable $\dot{p}$ is now a constant.

\section{Acknowledgements}

The financial support of Research Council of Zanjan University, Zanjan, Iran is appreciated. I also would like to thank Mr. B. Farnudi for editing the manuscript.

[1] Holland P.R., The quantum theory of motion, Cambridge University Press, 1993, 68-89.

[2] Sakurai J.J., Modern quantum mechanics, Addison-Wesley, 1985, 97-109.

[3] Madelung E., The hydrodynamical picture of quantum theory, Z. Phys., 1926, V.40, 322-326.

[4] Takabayasi T., The formulation of quantum mechanics in terms of ensemble in phase space, Progr. Theoret. Phys., 1954, V.11, 341-373.

[5] Muga J.G., Sala R., Snider R.F., Comparison of classical and quantum evolution of phase space distribution functions, Phys. Scripta, 1993, V.47, 732-739.

[6] Brown M.R., The quantum poptential: the breakdown of classical symplectic symmetry and the energy of localization and dispersion, quant-ph/9703007.

[7] Brown M.R., Hiley B.J., Schrödinger revisited: an algebraic approach, quant-ph/0005026.

[8] Bohm D., Hiley B.J., On the intuitive understanding of nonlocality and implied by quantum theory, Found. Phys., 1975, V.5, 93-109.

[9] Maroney O., Hiley B.J., Quantum teleportation understood through the Bohm interpretation, Found. Phys., 1999, V.29, 1403-1415.

[10] Bohm D., Hiley B.J., The undivided universe: the ontological interpretation of quantum theory, London, Routledge, 1993.

[11] Holland P.R., Quantum back-reaction and the particle law of motion, J. Phys. A: Math. Gen., 2006, V.39, 559-564.

[12] Shojai F., Shojai A., Constraints algebra and equation of motion in Bohmian interpretation of quantum gravity, Class. Quant. Grav., 2004, V.21, 1-9, gr-qc/0409035.

[13] Carroll R., Fluctuations, gravity, and the quantum potential, gr-qc/0501045.

[14] Carroll R., Some fundamental aspects of a quantum potential, quant-ph/0506075.

[15] Wigner E., On the quantum correction for thermodynamic equilibrium, Phys. Rev., 1932, V.40, 749-759.

[16] Moyal J.E., Quantum mechanics as a statistical theory, Proc. Camb. Philos. Soc., 1949, V.45, 99-124.

[17] Hillery M., O’Connell R.F., Scully M.O., Wigner E.P., Distribution functions in physics: fundamentals, Phys. Rep. C, 1984, V.106, 121-167. 
[18] Mehta C.L., Classical functions corresponding to given quantum operators, J. Phys. A, 1968, V.1, 385-392.

[19] Agarval G., Wolf E., Calculus for functions of noncommuting operators and general phase-space methods in quantum mechanics. I. Mapping theorems and ordering of functions of noncommuting operators, Phys. Rev. D, 1970, V.2, 2161-2186.

[20] Han D., Kim Y.S., Noz M.E., Linear canonical transformations of coherent and squeezed states in the Wigner phase space. II. Quantitative analysis, Phys. Rev. A, 1989, V.40, 902-912.

[21] Kim Y.S., Wigner E.P., Canonical transformation in quantum mechanics, Amer. J. Phys., 1990, V.58, 439-448.

[22] Jannussis A., Patargias N., Leodaris A., Phillippakis T., Streclas A., Papatheos V., Some remarks on the nonnegative quantum mechanical distribution functions, Preprint, Dept. Theor. Phys., Univ. of Patras, 1982.

[23] Bayen F., Flato M., Fronsdal C., Lichnerowicz A., Sternheimer D., Deformation theory and quantization, Ann. Physics, 1978, V.111, 61-110.

[24] Torres-Vega G., Ferderick J.H., Quantum mechanics in phase space: new approaches to the correspondence principle, J. Chem. Phys., 1990, V.93, 8862-8874.

[25] Torres-Vega G., Ferderick J.H., A quantum mechanical representation in phase space, J. Chem. Phys., 1993, V.98, 3103-3120.

[26] de Gosson M.A., Schrödinger equation in phase espace and deformation quantization, math.SG/0504013.

[27] Bolivar A.O., Quantum-classical correspondence dynamical quantization and the classical limit series: the frontiers collection, 2000.

[28] de Gosson M.A., The principles of Newtonian and quantum mechanics, the need for Planck's constant, $\hbar$, Blekinge Institute of Technology, Sweden, 2001.

[29] Sobouti Y., Nasiri S., A phase space formulation of quantum state functions, Internat. J. Modern Phys. B, 1993, V.7, 3255-3272.

[30] Goldstein H., Classical mechanics, London, Addison-Wesley, 1980.

[31] Merzbacher E., Quantum mechanics, New York, Wiley Interscience, 1970, 341-350. 